\title{
Calculation for the secular variation of the orbit of massive binary stars by the method of average values
}

\begin{abstract}
In this paper, the influence of the gravitational radiation damping on the evolution of the orbital elements of massive binary system is examined. The solutions are obtained by using the method of average values upon Gaussian perturbation equations. The theoretical results show that semi-major axis and eccentricity exhibit secular variation, but another orbital elements do not exhibit secular variation. The influence of the secular variation of the orbit would leads to collapse the system of binary stars. The theoretical results are applied to the calculation for the secular variation of the orbital elements of some known massive binary systems We concluded in this work that the gravitational radiation damping may results in that the orbital semi major axis of binary stars shrinks with time and the eccentricity decrease with time.
\end{abstract}

Volume 4 Issue 3 - 2020

\author{
Lin-Sen Li \\ School of physics, Northeast Normal University, China
}

Correspondence: Lin-Sen Li, School of physics, Northeast Normal University, Changchun, 130024, China, Email dbsd-s@@163.com

Received: October 30, 2019 | Published: June II, 2020

Keywords: gravitational radiation damping, massive binary systems, Gaussian perturbation equations, secular variation, orbital elements, eccentricity

\section{Introduction}

It is well known that the gravitational wave comes mainly from binary system..$^{1,2}$ Therefore, some binary systems are the best objects for testing the theory of the gravitational radiation. ${ }^{3}$ However, all binary systems are not suitable for the test. There are two possibilities for this test. One is two components with near separation: another one is two components with large mass. The former must be explored in the compact binary system. The latter is explored in the massive binary system. Lincoln et al. $^{4}$ research evolution and gravitational radiation of binary systems of compact objects to (post) ${ }^{5 / 2}$. Newtonian order. $\mathrm{Li}^{5}$ studied reaction effect of gravitational radiation of central body upon the variation of celestial orbital plane, but he do not studied the effect of gravitational radiation upon the orbital elements of celestial body. $\mathrm{Li}^{6}$ examined the gravitational radiation damping and evolution of the orbit of the compact binary stars by solution of the first perturbation method. $\mathrm{Li}^{7}$ studied influence of the gravitational radiation damping on the time of periastron passage of binary stars. $\mathrm{Li}^{8}$ also studied the gravitation radiation damping and the evolution of the orbit of compact binary stars by the solution of the second perturbation method. $\mathrm{Li}^{9}$ studied the secular effect of gravitational radiation damping on the periastron advance of binary stars in second order perturbation theory. However these methods are some trouble and expanding expression of the disturbing function is very long. If we only find the secular variation of the orbit, we may use the method of average to separate out the secular variable terms of the orbit and do not consider the periodic variable terms. Because it is important that find the secular variable term of orbit. This is advantage for the method of average. Because this paper only finds the secular variable terms of the orbital elements, so that this paper uses the method of average values to study the secular influence of gravitational radiation damping on the evolution of the orbit of massive binary stars.

\section{Solution by the method of average values upon Gaussian perturbation equations}

The formula for the relative acceleration with Post, Post-Post and Post $^{5 / 2}$ was given by Lincoln et al. ${ }^{4}$

$$
\vec{a}=\left(m / r^{2}\right)[(-1+A) \vec{n}+B \vec{V}] .
$$

For the gravitational emission, we take

$$
\vec{a}_{5 / 2}=\left(m / r^{2}\right)\left[\left(-1+A_{5 / 2}\right) \bar{n}+B_{5 / 2} \vec{V}\right] .
$$

Here $\mathrm{m}$ and $\mathrm{r}$ denote mass and separation of two binary stars respectively; $\vec{n}$ and $\vec{V}$ denote the unit vector of a radial direction and the vector of the relative velocity respectively. In the formula (2) the Newtonian term is $-\left(m / r^{2}\right) \vec{n}$ and the Post-Newtonain term (Post $)^{5 / 2}$ is $\left(m / r^{2}\right)\left(A_{5 / 2} \vec{n}+B_{5 / 2} \vec{V}\right)$

In the previous paper Li resolved the perturbation acceleration $\bar{a}_{5 / 2}$ into a radial component $\mathrm{R}_{5 / 2}$ and a transverse component $\mathrm{S}_{5 / 2}$ perpendicular to $\mathrm{R}_{5 / 2}$, and a component $\mathrm{W}_{5 / 2}$ normal to the orbital plane induced by gravitational radiation damping on the orbit of binary stars as follows $\mathrm{Li}^{9}$

$$
\begin{gathered}
R_{5 / 2}=\frac{8}{15} \eta\left(\frac{m}{r}\right)^{3}\left(\frac{m}{p}\right)^{\frac{1}{2}} p^{-1} e \sin f\left(14+6 e^{2}+20 e \cos f\right), \\
S_{5 / 2}=-\frac{8}{15} \eta \frac{m^{3}}{r^{4}}\left(\frac{m}{p}\right)^{\frac{1}{2}}\left(12+3 e^{2}+15 e \cos f\right), \\
\boldsymbol{W}_{5,2}=0 .
\end{gathered}
$$

Here $m=m_{1}+m_{2}, \quad \eta=\mu / m, \quad \mu=\frac{m_{1} m_{2}}{m},(\mathrm{G}=\mathrm{c}=1), f$ denotes the true anomaly. $p=a\left(1-e^{2}\right), a$ and $e$ denote semi-major axis 
and eccentricity respectively. Because this paper only researches the secular effect upon the evolution of the orbit, we use the average method. According to the definition of the average values, the average formula for the variable rate of the arbitrary orbital elements $\dot{\sigma}=F(a, e, \ldots \ldots .$.$) can be written as$

$$
\overline{\dot{\sigma}}=\frac{1}{T} \int_{0}^{T} F(a, e \ldots . . .) d t
$$

Here T is orbital period. The mean motion $n=\frac{2 \pi}{T}, d t=\frac{d M}{n} . M$ is the mean anomaly. The above average formula can be written as

$$
\overline{\dot{\sigma}}=\frac{1}{2 \pi} \int_{0}^{2 \pi} F(a, e,,,,,,) d M
$$

Gaussian equations can be written by average upon the mean anomaly $d M$ (Gaussian equations cite the symbol used by Lincoln et al. ${ }^{4}$

$$
\begin{gathered}
\overline{\dot{a}}=\frac{1}{2 \pi} \int_{0}^{2 \pi} \frac{2 a^{2}}{(m p)^{1 / 2}}\left[e R_{5 / 2} \sin f+(p / r) S_{5 / 2}\right] d M \\
=\frac{2 a^{2}}{(m p)^{1 / 2}}\left[e \overline{R_{5 / 2} \sin f}+\frac{\bar{p}}{r} S_{5 / 2}\right], \\
\overline{\dot{e}}=\frac{1}{2 \pi} \int_{0}^{2 \pi}(p / m)^{1 / 2}\left\{-R_{5 / 2} \sin f+\left[e\left(\frac{r}{p}\right)+\left(1+\frac{r}{p}\right) \cos f\right] S_{5 / 2}\right\} d M \\
=(p / m)^{1 / 2}\left[\overline{-R_{5 / 2} \sin f}+\overline{e(r / p)} S_{5 / 2}+\overline{\cos f} S_{5 / 2}+\frac{\left.\frac{r}{p} \cos f S_{5 / 2}\right],(7)}{\mathrm{e}^{\overline{\dot{\omega}}}=\frac{1}{2 \pi} \int_{0}^{2 \pi}{ }^{\left(\frac{p}{m}\right)}{ }^{1 / 2}\left[-R_{5 / 2} \cos f+\left(1+\frac{r}{p}\right) \sin f \cdot S_{5 / 2}\right] d M}\right. \\
=(p / m)^{1 / 2}\left[-\overline{R_{5 / 2} \cos f}+\overline{S_{5 / 2} \sin f}+\frac{\frac{r}{p} \sin f S_{5 / 2}}{p}-e\left(\frac{r}{p}\right) \cot i \sin f W_{5 / 2}\right] \\
\overline{\dot{i}}=\frac{1}{2} \int_{0-}^{2 \pi} \frac{r \cos f}{(m p)^{1 / 2} W_{5 / 2} d M},(8) \\
\overline{\dot{\Omega}}=\frac{1}{2 \pi} \int_{0}^{2 \pi} \frac{r \sin f}{(m p)^{1 / 2} \sin i} W_{5 / 2} d M,
\end{gathered}
$$

The longitude of periastron $\bar{\omega}$ in terms of the argument of periastron $\bar{\omega}$, can be written as

$$
\overline{\dot{\varpi}}=\overline{\dot{\omega}}+\bar{\Omega} .
$$

The equation of mean longitude of periastron at epoch $\varepsilon_{o}$ and the mean longitude $\lambda$ are $^{10}$

$$
\overline{\dot{\varepsilon}_{o}}=-\frac{2 \overline{R_{5 / 2} r}}{n a^{2}}+\frac{e^{2}}{1+\left(1-e^{2}\right)^{1 / 2}} \overline{\dot{\omega}},
$$

$\overline{\dot{\lambda}}=n+\overline{\dot{\varepsilon}_{0}} \cdot$ (13) Next, we take the average values of each term in the Gaussian equation according to the formulae of the average values of some functions given by $\mathrm{Liu}^{11}$

$$
\overline{\left(\frac{a}{r}\right)^{p} \sin q f,}: \overline{\left(\frac{a}{r}\right)^{p} \cos q f},(\mathrm{p}, \mathrm{q}=1,2,3) .
$$

The average values used in this paper are list in Appendix.: A(a), A(b), A(c), A(d), A(e), A(f), Ag), A(h), A(i), A(j), A(k).

\section{Appendix}

Same orbital average results are chose from the Table given by Liu (1992) which are cited in this paper.

$$
\begin{gathered}
\overline{\left(\frac{a}{r}\right)^{3}=\left(1-e^{2}\right)^{-3 / 2}, \quad \mathrm{~A}(\mathrm{a}) \overline{\left(\frac{a}{r}\right)^{3} \cos f}}=\frac{1}{2} e\left(1-e^{2}\right)^{-3 / 2} \\
\overline{\left(\frac{a}{r}\right)^{3} \cos 2 f}=0 \quad \mathrm{~A}(\mathrm{c}) \quad \overline{\left(\frac{a}{r}\right)^{3} \cos 3 f}=0 \\
\end{gathered}
$$

$\overline{\left(\frac{a}{r}\right)^{4} \cos f}=e\left(1-e^{2}\right)^{-5 / 2}, \quad \overline{\mathrm{A}(\mathrm{f})} \overline{\left(\frac{a}{r}\right)^{4} \cos 2 f}=\frac{1}{4} e^{2}\left(1-e^{2}\right)^{-5 / 2}$,

$$
\left(\frac{\bar{a}}{r}\right)^{5}=\left(1+\frac{3}{2} e^{2}\right)\left(1-e^{2}\right)^{-7 / 2}
$$

$$
\overline{\left(\frac{a}{r}\right)^{5} \cos f=\frac{3}{2}} e\left(1+\frac{1}{4} e^{2}\right)\left(1-e^{2}\right)^{-7 / 2}
$$

$$
\begin{aligned}
& \overline{\left(\frac{a}{r}\right) \sin f}=\overline{\left(\frac{a}{r}\right)^{2} \sin f}=\overline{\left(\frac{a}{r}\right)^{3} \sin f}=\overline{\left(\frac{a}{r}\right)^{4} \sin f=0} \\
& \left(\frac{a}{r}\right)^{2} \sin 2 f=\overline{\left(\frac{a}{r}\right)^{3} \sin 2 f}=\overline{\left(\frac{a}{r}\right)^{3} \sin 3 f}=\overline{\left(\frac{a}{r}\right)^{4} \sin 2 f=0 .}
\end{aligned}
$$

Using the following average terms with $\mathrm{R}_{5 / 2}$ given by the formula (3), we yield

$$
\begin{aligned}
& \because \overline{R_{5 / 2} \sin f}=\frac{1}{2 \pi} \int_{0}^{2 \pi} \frac{8}{15} \eta\left(\frac{m}{r}\right){ }^{3}\left(\frac{m}{p}\right)^{1 / 2} p^{-1} e \sin ^{2} f\left(14+6 e^{2}+20 e \cos f\right) d M \\
& , \overline{R_{5 / 2} \sin f}=\frac{8}{15} \eta\left(\frac{m}{a}\right)^{3}\left(\frac{m}{p}\right)^{1 / 2} p^{-1}\left(\frac{a}{r}\right)^{3}\left[\left(7 e+3 e^{3}\right)+\frac{15}{2} e^{2} \cos f-\left(7 e+3 e^{3}\right) \cos 2 f-\frac{5}{2} e^{2} \cos 3 f\right]
\end{aligned}
$$

Substitution of the following A(a), A(b), A(c) and A(d) into the above expression

$\overline{\left(\frac{a}{r}\right)^{3}}=\left(1-e^{2}\right)^{-3 / 2}, \overline{\left(\frac{a}{r}\right)^{3} \cos f}=\frac{1}{2} e\left(1-e^{2}\right)^{-3 / 2}, \overline{\left(\frac{a}{r}\right)^{3} \cos 2 f}=0, \overline{\left(\frac{a}{r}\right)^{3} \cos 3 f}=0$, $1 / a^{3}=p^{-3}\left(1-e^{2}\right)^{3}$,

We obtain

$$
\therefore \overline{R_{5 / 2} \sin f}=\frac{8}{15} \eta m^{7 / 2} p^{-9 / 2}\left(1-e^{2}\right)^{3 / 2}\left(7 e+\frac{11}{2} e^{3}\right) \text {. }
$$

$$
\begin{aligned}
\because \overline{R_{5 / 2} \cos f}=\frac{1}{2 \pi} \int_{0}^{2 \pi} \frac{8}{15} \eta\left(\frac{m}{r}\right)^{3}\left(\frac{m}{p}\right)^{1 / 2} p^{-1} e \sin f \cos f\left(14+6 e^{2}+20 e \cos f\right) d M \\
=\frac{8}{15} \eta\left(\frac{m}{p}\right)^{3}\left(\frac{m}{p}\right)^{1 / 2} p^{-1}\left(1-e^{2}\right)^{3}\left(\frac{a}{r}\right)^{3}\left[\left(7 e+3 e^{3}\right) \sin 2 f\right. \\
\left.+5 e^{2} \sin f+5 e^{2} \sin 3 f\right] .
\end{aligned}
$$

Substitution of the third formula of $\mathrm{A}(\mathrm{j})$ and the second and third formulas of $\mathrm{A}(\mathrm{k})$ into the above expression

$$
\left.\overline{\left(\frac{a}{r}\right.}\right)^{3} \overline{\sin f}=0, \overline{\left(\frac{a}{r}\right)^{3} \sin 2 f}=0, \overline{\left(\frac{a}{r}\right)^{3}} \sin 3 f=0,
$$


We obtain

$$
\therefore \overline{R_{5 / 2} \cos f}=0 \text {. }
$$

Next, using the following averages with $\mathrm{S}_{5 / 2}$ given by using (4), we get

$\because \overline{\left(\frac{p}{r}\right) S_{5 / 2}}=-\frac{1}{2 \pi} \int_{0}^{2 \pi} \frac{8}{15} \frac{m^{3}}{a^{5}}\left(\frac{m}{p}\right)^{1 / 2} p \cdot\left(\frac{a}{r}\right)^{5}\left[\left(12+3 e^{2}\right)+15 e \cos f\right] d M$,

$$
\overline{(p / r) S}_{5 / 2=-} \frac{8}{15} m^{3} m^{1 / 2} p^{-5} p\left(1-e^{2}\right)^{5} \overline{\left(\frac{a}{r}\right)^{5}}\left[\left(12+3 e^{2}\right)+15 e \cos f\right]
$$

Substitution of the following $\mathrm{A}(\mathrm{h})$ and $\mathrm{A}(\mathrm{i})$ into the above expression ,

$$
\overline{\left(\frac{a}{r}\right)^{5}}=\left(1+\frac{3}{2} e^{2}\right)\left(1-e^{2}\right)^{-7 / 2}, \overline{\left(\frac{a}{r}\right)^{5} \cos f}=\frac{3}{2} e\left(1+\frac{1}{4} e^{2}\right)\left(1-e^{2}\right)^{-7 / 2},
$$

We obtain

$\therefore \overline{\left(\frac{p}{r}\right) S_{5 / 2}}=-\frac{8}{15} \eta m^{7 / 2} p^{-9 / 2}\left(1-e^{2}\right)^{3 / 2}\left(12+\frac{87}{2} e^{2}\right)$.

$\Theta \overline{e\left(\frac{r}{p}\right) S_{5 / 2}}=-\frac{1}{2 \pi} \int_{0}^{2 \pi} \frac{8}{15} \eta m^{1 / 2} m^{3} p^{-1 / 2} p^{-1} a^{-3} e\left(\frac{a}{r}\right)^{3}\left(12+3 e^{2}+15 e \cos f\right) d M$

$$
=-\frac{8}{15} \eta m^{7 / 2} p^{-9 / 2}\left(1-e^{2}\right)^{3}\left(\frac{\bar{a}}{r}\right)^{3}\left[\left(12 e+3 e^{3}\right)+15 e^{2} \cos f\right]
$$

Substitution of the following A(a) , A(b) into the above expression, $\overline{(a / r)^{3}}=\left(1-e^{2}\right)^{-3 / 2}, \overline{\left(\frac{a}{r}\right)^{3} \cos f}=\frac{1}{2} e\left(1-e^{2}\right)^{-3 / 2}$,

We obtain

$\therefore \overline{e\left(\frac{r}{p}\right) S_{5 / 2}}=-\frac{8}{15} \eta m^{7 / 2} p^{-9 / 2}\left(1-e^{2}\right)^{3 / 2}\left(12 e+\frac{21}{2} e^{3}\right)$.

$\left.\because \overline{S_{5 / 1} \cos f}=-\frac{1}{2 \pi} \int_{0}^{2 \pi} \frac{8}{15} \eta \frac{m^{3}}{r^{4}}\left(\frac{m}{p}\right)^{1 / 2} \cos f\left(12+3 e^{2}\right)+15 e \cos f\right) d M$ $\overrightarrow{S_{5 / 2} \cos f}=\frac{8}{15} \eta m^{7 / 2} p^{-9 / 2}\left(1-e^{2}\right)^{4} \overline{\left(\frac{a}{r}\right)^{4}}\left[\left(12+3 e^{2}\right) \cos f+\frac{15}{2} e \cos 2 f+\frac{15}{2} e\right]$

Substitution of the following $\mathrm{A}(\mathrm{f}), \mathrm{A}(\mathrm{g})$ and $\mathrm{A}(\mathrm{e})$ into the above expression

$\overline{\mathrm{c}} \overline{S_{5 / 2} \cos f}=\frac{8}{15} \eta m^{7 / 2} p^{-9 / 2}\left(1-e^{2}\right)^{4} \overline{\left(\frac{a}{r}\right)^{4}}\left[\left(12+3 e^{2}\right) \cos f+\frac{15}{2} e \cos 2 f+\frac{15}{2} e\right]$

We obtain

$$
\therefore \overline{S_{5 / 2} \cos f}=-\frac{8}{15} \eta m^{7 / 2} p^{-9 / 2}\left(1-e^{2}\right)^{3 / 2}\left(\frac{39}{2} e+\frac{69}{8} e^{3}\right)
$$

$\because \overline{\frac{r}{p} \cos f S_{5 / 2}}=-\frac{1}{2 \pi} \int_{0}^{2 \pi} \frac{8}{15} \eta \frac{m^{3}}{r^{3}} m^{1 / 2} p^{-1} \cos f\left[\left(12+3 e^{2}\right)+15 e \cos f\right] d M$,

$\overline{\frac{r}{p} \cos f S_{5 / 2}}=-\frac{8}{15} \eta m^{7 / 2} p^{-9 / 2}\left(1-e^{2}\right)^{3} \overline{\left(\frac{a}{r}\right)^{3}}\left[\left(12+3 e^{2}\right) \cos f+\frac{15}{2} e+\frac{15}{2} e \cos 2 f\right]$

Substitution of the following $\mathrm{A}(\mathrm{b}) \mathrm{A}(\mathrm{a})$ and $\mathrm{A}(\mathrm{c})$ into the above expression $\phi \overline{\left(\frac{a}{r}\right)^{3}}=\left(1-e^{2}\right)^{-3 / 2}, \overline{\left(\frac{a}{r}\right)^{3} \cos f}=\frac{1}{2} e(1-e)^{-3 / 2}, \overline{\left(\frac{a}{r}\right)^{3} \cos 2 f}=0$,

We obtain

$\therefore \overline{\frac{r}{p} \cos f S_{5 / 2}}=-\frac{8}{15} \eta m^{7 / 2} p^{-9 / 2}\left(1-e^{2}\right)^{3 / 2}\left(\frac{27}{2} e+\frac{3}{2} e^{3}\right)$

$\because \overline{S_{5 / 2} \sin f}=-\frac{1}{2 \pi} \int_{0}^{2 \pi} \frac{8}{15} \frac{m^{3}}{r^{4}}\left(\frac{m}{p}\right)^{1 / 2} \sin f\left[\left(12+3 e^{2}\right)+15 e \cos f\right] d M ，$

$\overline{S_{5 / 2} \sin f}=-\frac{8}{15} m^{7 / 2} p^{-9 / 2}\left(1-e^{2}\right)^{4}\left(\overline{\left.\frac{a}{r}\right)^{4}}\left[\left(12+3 e^{2}\right) \sin f+\frac{15}{2} e \sin 2 f\right]\right.$,

Substitution of the following $A(j)$ and $A(k)$ into the above expression

$$
\overline{\left(\frac{a}{r}\right)^{4} \sin f}=0, \overline{\left(\frac{a}{r}\right)^{4} \sin 2 f}=0
$$

We obtain

$$
\therefore \overline{S_{5 / 2} \sin f}=0 \text {. }
$$

$\because \overline{\frac{r}{p} \sin f S_{5 / 2}}=-\frac{1}{2 \pi} \int_{0}^{2 \pi} \frac{8}{15} \frac{m^{3}}{r^{3}} m^{1 / 2} p^{-1 / 2} p^{-1} \sin f\left[\left(12+3 e_{2}\right)+15 e \cos f\right] d M$ $\overline{\frac{r}{p} \sin f S_{5 / 2}}=\frac{8}{15} \eta m^{7 / 2} p^{-9 / 2}\left(1-e^{2}\right)^{3} \overline{\left(\frac{a}{r}\right)^{3}}\left[\left(12+3 e^{2}\right) \sin f+\frac{15}{2} e \sin 2 f\right]$

Substitution of the following third formula $A(j)$ and second formula of $\mathrm{A}(\mathrm{k})$ into the above expression

$$
\because \overline{\left(\frac{a}{r}\right) \sin f}=0, \overline{\left(\frac{a}{r}\right)^{3} \sin 2 f}=0,
$$

We obtain

$$
\therefore \overline{\frac{r}{p} \sin f \cdot S_{5 / 2}}=0 \text {. }
$$

$\because \overline{r R}_{5 / 2}=-\frac{1}{2 \pi} \int_{0}^{2 \pi} \frac{8}{15} \eta \frac{m^{3}}{r^{2}} m^{1 / 2} p^{-1 / 2} p^{-1} e \sin f\left(4+6 e^{2}+20 e \cos f\right) d M$

$$
=\frac{8}{15} \eta m^{7 / 2} p^{-7 / 2}\left(1-e^{2}\right)^{2} e \overline{\left(\frac{a}{r}\right)^{2}}\left[\left(4+6 e^{2}\right) \sin f+10 e \sin 2 f\right]
$$

Substitution of the following second formula and first formula of $\mathrm{A}(\mathrm{k})$ into the above expression,

$$
\overline{\left(\frac{a}{r}\right)^{2} \sin f}=0, \overline{\left(\frac{a}{r}\right)^{2} \sin 2 f}=0,
$$

We obtain

$$
\therefore \overline{r R}_{5 / 2}=0 \quad \overline{W_{5 / 2}}=0
$$

Substituting formulae (14) and (17) into the equation (6), we obtain

$$
\overline{\dot{a}}-\frac{8}{15} \eta\left(\frac{m}{p}\right)^{3}\left(1-e^{2}\right)^{-1 / 2}\left(24+73 e^{2}\right) .
$$

Substituting the formulae (14) and (16) into the equation (7), we obtain

$$
\overline{\dot{e}}=-\frac{8}{15} \eta\left(\frac{m}{p}\right)^{3} p^{-1}\left(1-e^{2}\right)^{3 / 2}\left(38 e+\frac{121}{8} e^{3}\right) .
$$


Substituting the formulae (15) and (21)-(22) into the equation (8), we obtain

$$
\overline{\dot{\omega}}=0
$$

Substituting the formula (5) or $W_{5 / 2}=0$ into the equation (9)-(10), we obtain

$$
\begin{gathered}
\bar{i}=\bar{\Omega}=0, \\
\overline{\dot{\varpi}}=\overline{\dot{\omega}}+\overline{\dot{\Omega}}=0 .
\end{gathered}
$$

Substituting the formulae (22) and (25) into the equation (12), we obtain

$$
\dot{\varepsilon}_{0}=0 \text {. }
$$

According to the formulae (13) and (28)

$$
\overline{\dot{\lambda}}=n+\overline{\dot{\varepsilon}_{0}} \text {. }
$$

The variable rate of the period $\mathrm{P}$ is given by the Kepler's third law and the equation (23)

$$
\overline{\dot{P}}=\frac{3}{2}\left\{\frac{P}{a}\right) \frac{\overline{d a}}{d t} .=-\frac{4}{5} \eta\left(\frac{m^{3}}{p^{4}}\right)\left(1-e^{2}\right)^{-3 / 2}\left(24+73 e^{2}\right) P(s / c y) .
$$

Here P denotes the orbital period.

The lifetime (spiral time)

$$
\tau=\frac{a}{\dot{a}}(y r)
$$

\section{Numerical results}

We use the formulae (23)-(30) to calculate the secular influence of gravitational radiation damping upon the orbital elements of four massive binary systems., but it is necessary to reduce the formulas (23)- (30) as an applicable formulae before calculation. As in the section 2, the right hand of the formulas (3)-(5) need to be multiplied by $1 / \mathrm{c}^{2}$, and $m$ should be multiplied by $\mathrm{G}$

$$
\begin{aligned}
\eta m^{3} & =\frac{m_{1} m_{2}}{m^{2}} m^{3}=m_{1} m_{2} m=G^{3} m_{1} m_{2}\left(m_{1}+m_{2}\right), \\
\therefore \quad \eta m^{2} & =m_{1} m_{2}=G^{2} m_{1} m_{2}, p=a\left(1-e^{2}\right) .
\end{aligned}
$$

The formulae (23)-(30) become

$$
\begin{aligned}
& \overline{\dot{a}}=-\frac{8}{15} \pi\left(\frac{G^{3}}{c^{5}}\right) \frac{m_{1} m_{2}\left(m_{1}+m_{2}\right)}{a^{3}\left(1-e^{2}\right)^{7 / 2}}\left(24+73 e^{2}\right), \\
& \overline{\dot{e}}=-\frac{8}{15} \pi\left(\frac{G^{3}}{c^{5}}\right) \frac{m_{1} m_{2}\left(m_{1}+m_{2}\right)}{a^{4}\left(1-e^{2}\right)^{5 / 2}} e\left(38+\frac{121}{8} e^{2}\right), \\
& \overline{\dot{\omega}}==\overline{\dot{\omega}}=0, \\
& \overline{\dot{\varepsilon}_{0}}=0, \\
& \overline{\dot{P}}=\frac{3}{2}\left(\frac{P}{a}\right) \overline{\dot{a}} .=-\frac{4}{5} \frac{G^{3}}{c^{5}} \frac{m_{1} m_{w}\left(m_{1}+m_{2}\right)\left(24+73 e^{2}\right)}{a^{4}\left(1-e^{2}\right)^{7 / 2}} P .
\end{aligned}
$$

It is convenient that $\mathrm{m}_{1}, \mathrm{~m}_{2}$ and $a$ are denoted by the unit in solar mass $\mathrm{M}\left(\mathrm{M}_{\odot}\right), \mathrm{M}_{\odot}=1.989 \times 10^{33} \mathrm{~g}$ and solar radius a $\left(\mathrm{R}_{\odot}\right)$, $\mathrm{R}_{\odot}=6.9599 \times 10^{10} \mathrm{~cm}, P$ is denotes by the unit in day $=86400 \mathrm{~s}$ $\mathrm{G}=6.67 \times 10^{-8}(\mathrm{c}, \mathrm{g}, \mathrm{s}), \mathrm{c}=3 \times 10^{10} \mathrm{~cm} / \mathrm{s}$. Substituting these data into the formulae (32), we get

$$
\begin{aligned}
& \overline{\dot{a}}=-4.8675 \frac{M_{1} M_{2}\left(M_{1}+M_{2}\right)\left(24+73 e^{2}\right)}{A^{3}\left(1-e^{2}\right)^{7 / 2}}(\mathrm{~cm} / y r), \\
& \overline{\dot{e}}=-6.8513 \times 10^{-11} \frac{M_{1} M_{2}\left(M_{1}+M_{2}\right)}{A^{4}\left(1-e^{2}\right)^{5 / 2}} e\left(38+\frac{121}{8} e^{2}\right) / y r, \\
& \overline{\dot{\omega}}=\overline{\bar{\varpi}}=0, \\
& \overline{\dot{\varepsilon}_{0}}=0, \\
& \overline{\dot{P}}=-9.314 \times 10^{-6} \frac{M_{1} M_{2}\left(M_{1}+M_{2}\right)\left(24+73 e^{2}\right)}{A^{4}\left(1-e^{2}\right)^{7 / 2}} \cdot P(s / y r) .
\end{aligned}
$$

\begin{tabular}{|c|c|c|c|c|}
\hline Binary stars & Y Cyg & V382 Cyg & V448Cyg & $\delta$ Ori \\
\hline$\dot{a}(\boldsymbol{m} / y)$ & -56.5 & -635.9 & -13.13 & -139.96 \\
\hline$\dot{e}\left(\mathbb{Q}^{-\mathrm{D}} / \not y\right)$ & -6.5 & -21.3 & -0.038 & -0.11 \\
\hline$\dot{\omega}=\dot{\varpi}(\mathrm{rad} / y)$ & 0 & 0 & 0 & 0 \\
\hline$\dot{\varepsilon}_{0}(\mathrm{rad} / \mathrm{y})$ & 0 & 0 & 0 & 0 \\
\hline$\dot{\lambda}(\mathrm{rad} / \mathrm{y})$ & 766 & $1212 \mid$ & 348 & 400 \\
\hline$\dot{P}\left(\mathbb{0}{ }^{-5} s / y\right)$ & -1.1 & -8.4 & -0.35 & -0.34 \\
\hline$t\left(\mathbb{l}^{9} y\right)$ & 35 & 2.9 & 265 & 22.87 \\
\hline
\end{tabular}

This paper chooses four massive binary stars V382 Cyg, V448 Cyg, Y Cyg, ö Ori as an example. For these binary stars, their data for $\mathrm{P}(\mathrm{d}), \mathrm{A}\left(\mathrm{R}_{\odot}\right), \mathrm{M}_{1}\left(\mathrm{M}_{\odot}\right), \mathrm{M}_{2}\left(\mathrm{M}_{\odot}\right)$ and $e$ are cited from the references listed in Table 1. Substituting these data for P (d), $A\left(R_{\Theta}\right), M_{1}\left(M_{\Theta}\right)$ , $M_{2}\left(M_{\Theta}\right)$ and $e$ of four massive binary stars in Table 1 into the formulas (33), we obtain the numerical results for the secular effect of gravitational radiation damping upon the orbital elements of four massive binary systems listed in Table 2 .

Table I Data of four massive binary systems

\begin{tabular}{lllllll}
\hline $\begin{array}{l}\text { Massive } \\
\text { binary } \\
\text { stars }\end{array}$ & $\mathbf{P}(\mathbf{d})$ & $\begin{array}{l}A(R \\
\odot\end{array}$ & $\begin{array}{l}M_{1}(M \\
\odot\end{array}$ & \multicolumn{2}{c}{$M_{2}\left(M_{\odot}\right)$} & References \\
\hline Y Cyg & 2.9963 & 28.49 & 17.57 & 17.04 & 0.1415 & Simon et al' \\
$\begin{array}{l}\text { Y } 382 \\
\text { Cyg }\end{array}$ & 1.8855 & 26.44 & 37.16 & 36.42 & 0.04 & Brancewicz $^{13}$ \\
Y448Cyg & 6.5797 & 50.09 & 23.84 & 15.73 & 0.04 & $\begin{array}{l}\text { Batten } \\
\text { 12 }\end{array}$ \\
$\delta$ Ori & 5.7324 & 45.99 & 30.57 & 9.17 & 0.1 & $\begin{array}{l}\text { Batten et } \\
\text { al' }\end{array}$ \\
\hline
\end{tabular}

Table 2 Numerical results for the secular effects of gravitational radiation upon the orbital elements of four massive binary stars

\section{Discussion}

\section{The comparison of the present paper with the previous} paper

In the previous paper ${ }^{8}$ there are both secular and periodic variation for semi-major axis and eccentricity.. However, it is very long and trouble for expanding perturbation function by using the perturbation 
method. In the present paper there is only secular variation for semimajor axis and eccentricity, and there is not periodic variation if we do not consider the periodic variation. However, it is a simple method for deriving the orbital elements by using a method of average values. The numerical results show that the orbital effects of massive binary stars are larger than that of the compact binary stars in some cases. For example, in the previous paper the variable rate of semi-major axis $\frac{d a}{d t}=-143(\mathrm{~cm} / \mathrm{yr})$ for the compact binary system PSR1913+16: but in the present paper $\frac{d a}{d t}=-635(\mathrm{~cm} / \mathrm{yr})$ for the massive binary system V382 Cyg So that the value of the orbital effect of massive binary star is large than that of the compact binary star. However, it is not all massive binary stars that their orbital effect is large, and only the massive binary stars with smaller separation are such case. For example, in the present paper for the massive binary star V448 Cyg even through their masses are large, but their orbital effect $\frac{d a}{d t}=-13.13(\mathrm{~cm} / y r)$ is small because their separation is large.

The theoretical results obtained by using the average method are consistent with the theoretical results obtained by using the perturbation method in the previous paper even through the forms of solutions are not different. However, both theoretical results may be transformed mutually in both methods. For example in the previous work $^{8}$ the secular variable of the semi-major axis is

$$
\Delta a=2 \pi A_{0}=2 \pi\left\{\frac{8}{15} \eta m^{5 / 2} p^{-3 / 2}\left(1-e^{2}\right)^{-2}\left(24+73 e^{2}\right) .\right\}
$$

We can write this formula divide by the period $\mathrm{T}$

$$
\overline{\dot{a}}=\frac{\Delta a}{T}=\frac{2 \pi}{T}\left\{\frac{8}{15} \eta m^{5 / 2} p^{-3 / 2}\left(1-e^{2}\right)^{-2}\left(24+73 e^{2}\right) .\right\} .
$$

Using Kepler's third law $\frac{2 \pi}{T}=n=\frac{m^{1 / 2}}{a^{3 / 2}}, \quad p=a\left(1-e^{2}\right)$.

Substituting these into the above expression, we can transform to the formula (32)

$$
\overline{\dot{a}}=-\frac{8}{15} \eta\left(\frac{m}{p}\right)^{3}\left(1-e^{2}\right)^{-1 / 2}\left(24+73 e^{2}\right)
$$

This means that the results in this paper are all correct with previous paper and may be transformed mutually.

\section{The comparison of this work with the work of other author}

In this paper although the derivation for the formulae ((1)-(3) based on the work of Lincoln et al. ${ }^{4}$ however, the derivation in both method are different. The present paper obtained the secular effect on the orbital elements by using a method of average values. The work of L-W obtained the secular effect of the orbital elements by using the iterative method. However, the results obtained by both methods may be transformed to the same results for semi-major axis and eccentricity through the following relative formula:

$$
\Delta a=(\Delta p+2 a e) /\left(1-e^{2}\right) . \quad p=a\left(1-e^{2}\right) .
$$

We can obtain from the results (3.1a) and (3.1b) of the works of Lincoln[-Will

$$
\Delta a=2 \pi\left\{\frac{8}{15} \eta m^{5 / 2} p^{-3 / 2}\left(1-e^{2}\right)^{-2}\left(24+73 e^{2}\right) .\right\}
$$

This expression may be transformed to the formula (32) in this paper

$$
\overline{\dot{a}}=-\frac{8}{15} \eta\left(\frac{m}{p}\right)^{3}\left(1-e^{2}\right)^{-1 / 2}\left(24+73 e^{2}\right) .
$$

The possibility of the observed effect It is possible to observe the effect of gravitational radiation damping on the orbit of massive binary stars because as in the calculated results for the massive binary star V382 Cyg, its orbital semi-major axis shrinks over $6.3 \mathrm{~m}$ per year, such large effect may be observed by using the recent astronomical telescope through per year. ${ }^{10-17}$

\section{Conclusion}

We conclude that the gravitational radiation damping may results in that the orbital semi major axis of binary stars shrinks with time and the eccentricity decrease with time. It can be seen from the numerical results in Table 2 that for the lifetime (spiral time) of four massive binary systems the longest lifetime is the order $10^{11}$ year for V448 Cyg. The smallest lifetime is the order $10^{9}$ year for V382Cyg. Another both massive binary stars are the order $10^{10}$ year. So that the first collapsed binary star is the massive star V382 Cyg. The last collapsible massive binary star is V448 Cyg. The collapse time of these binary systems are very meaningful. Because when systems collapse and two massive binary stars collide mutually, the gravitational wave will be emitted possibly.

We also conclude that we may use different methods to research the same topic. If we consider secular and periodic variation of orbit, the best method is used to the perturbation method: if we only consider secular effect of the orbit, the best method is used to the method of average values.

For observing the effect of the gravitation emission damping on the orbit, the best means may explore in the compact binary system and also may explore in the massive binary system. However, it is not all massive binary system that their orbital effects are large, only the massive binary system with smaller reparation is such case.

\section{Acknowledgments}

None.

\section{Conflicts of interest}

The author declares there is no conflict of interest.

\section{Funding}

None.

\section{References}

1. Weinberg S. Gravitation and Cosmology: Principles and Applications to the General Theory of Relativity. Chapter 10 Gravitation radiation, 287, John Wiley, New York, 1972.

2. Misner CW, Thorne KS, Wheeler JA. Gravitation, Part 111 Gravitational waves, 941, W H Freeman, San Francisco U S. 1973.

3. Arras P. Gravitational waves and the maximum spin rate of accreting neutron stars. ASP Conference Series Vol 382 edited by FA Rasio \& IH Stairs. 2005. p. 317

4. Lincoln $\mathrm{CW}$, Will $\mathrm{CM}$. Coalescing binary systems of compact objects to (Post $)^{5 / 2 \ldots}$ Newtonian order: Late-time evolution and gravitational-radiation emission. Phys Rev. 1990;D42(4):1123. 
5. Li Lin-Sen. Reaction effect of gravitational radiation of rotating ellipsoid as central body upon the variation of celestial orbital plane. Chinese Physics Letters. 1997;14(5):328.

6. Li Lin-Sen. Gravitational radiation damping and evolution of the orbit of compact binary pulsars (Solution by the first perturbation method). IL NUOVO CIMENTO. 2009;124B(7):709.

7. Li Lin-Sen. Influence of the gravitational radiation damping on the time of periastron passage of binary stars. Astrophys \& Space Sci. 2011;334:125.

8. Li Lin-Sen. Gravitational radiation damping and evolution of the orbit of compact binary stars (Solution by the second perturbation method). $J$ Astrophys Astronomy. 2014;35(2):189.

9. Li Lin-Sen. The secular effect of gravitational radiation damping on the periastron advance of binary stars in second order perturbation theory. Res Astron Astrophys. 2017;17(8):84.

10. Brouwer D, Clemence GM. Method of Celestial Mechanics. $1^{\text {st }}$ ed. Academic Press, New York and London. 1961.

11. Liu Lin. Orbital Mechanics for Artificial Earth Satellite. High Education Press, Beijing, 1992. p. 566.
12. Batten AH, Flecher JM, MacCarthy DG. Eighth Catalogue of the Orbital Elements of Spectroscopic Binary Systems, Publ. Dominion. Astrophys Obs. 1989;17(1).

13. Brancewicz HK, Dwornk TZ. A Catalogue of Parameters for Eclipsing Binaries. Acta Astron. 1980;30:501.

14. Damour T, Gopakumar A, Iyer BR. Phasing of gravitational waves from inspiralling eccentric binaries. General Relativity and Quantum Cosmology. 2004;30:1-49.

15. Simon KP, Sturm E, Fiedler A. Spectroscopic analysis of hot binaries II the components of Y Cygni. Astron Astrophys. 1994;292:507.

16. Will CW. Theory and experiment in gravitational physics. Chapter 10 Gravitational Radiation as a tool for testing relativistic gravity, 221, Cambridge University Press, Cambridge, U K, New York, US. 1993.

17. Will CM. The Confrontation between General Relativity and Experiment, 4 Strong Gravity and Gravitational Waves: New texting ground, Living Rev. Relativity. 2006;9:3. 